\title{
Validation of blood culture gram staining for the detection of Staphylococcus aureus by the 'oozing sign' surrounding clustered gram-positive cocci: a prospective observational study
}

\author{
Yoshiro Hadano ${ }^{1,2^{*}}$, Miwako Isoda ${ }^{3}$, Kazushige Ishibashi ${ }^{3}$ and Tatsuyuki Kakuma²
}

\begin{abstract}
Background: Staphylococcus aureus bacteraemia is a common and significant infection, associated with high rates of mortality. Therefore, early identification is important for the initiation of appropriate treatment. The objective of this study was to evaluate the accuracy of blood culture Gram staining along with the finding of an 'oozing sign' to diagnose either Staphylococcus aureus or coagulase-negative staphylococci.
\end{abstract}

Methods: This single-centre, prospective observational study was performed from May 2017 to November 2017. We used routine blood culture bottles (BacT/ALERT FA and BacT/ALERT SN; bioMérieux, Inc., Durham, NC). Bacterial species were identified and the minimum inhibitory concentration was determined by using the MicroScan WalkAway 96 SI system (Beckman Coulter, Tokyo, Japan). Bottles showing growth were removed, and Gram staining was performed.

Results: A total of 118 samples, including 55 aerobic and 63 anaerobic bottle samples, were analysed. The overall sensitivity of Gram staining was 78.7\% (95\% Cl: 65.8-94.3\%), and the specificity was 95.0\% (95\% Cl: 84.7-98.4\%).

Conclusion: The 'oozing sign' observed in Gram staining may be useful for the rapid prediction of $S$. aureus in BacT/ALERT blood culture bottles.

Keywords: Staphylococcus aureus, Gram staining, 'Oozing', BacT/ALERT blood culture bottles

\section{Background}

Staphylococcus aureus bacteraemia (SAB) is a common, significant infection. The 30-day all-cause mortality for SAB is $20-30 \%$, and this has not changed since the 1990 s [1]. Early identification is important for quickly initiating an appropriate treatment to prevent persistent bacteraemia, which is associated with a worse outcome [2]. Recently, a new, rapid and reliable identification system, matrix-assisted laser desorption/ionization time-of-flight mass spectrometry (MALDI-TOS MS), was introduced for the diagnosis of microbial pathogens [3]. However,

\footnotetext{
* Correspondence: hatayoshiyoshi@gmail.com

'Department of Infectious Diseases, St. Mary's Hospital, Kurume, Japan

${ }^{2}$ Biostatistics Center, Kurume University School of Medicine, Kurume, Japan Full list of author information is available at the end of the article
}

most community hospitals do not have the necessary equipment to perform MALDI-TOS MS due to the high cost of these systems. S. aureus is most commonly identified in culture by using the coagulase test, and confirmation requires an additional $24 \mathrm{~h}$ [4].

Gram staining is a classical method that is convenient and provides considerable information in a short period of time. In our daily experience, a finding of a pink-coloured 'oozing' component, which we have termed an 'oozing sign', surrounding the clustered gram-positive cocci is sometimes observed for S. aureus but for other staphylococci (Fig. 1). Thus, we hypothesized that this 'oozing sign' may be a key diagnostic finding that can be used to distinguish S. aureus from other staphylococci. The objective of this study was to evaluate the accuracy of Gram staining

(c) The Author(s). 2018 Open Access This article is distributed under the terms of the Creative Commons Attribution 4.0 International License (http://creativecommons.org/licenses/by/4.0/), which permits unrestricted use, distribution, and reproduction in any medium, provided you give appropriate credit to the original author(s) and the source, provide a link to the Creative Commons license, and indicate if changes were made. The Creative Commons Public Domain Dedication waiver (http://creativecommons.org/publicdomain/zero/1.0/) applies to the data made available in this article, unless otherwise stated. 


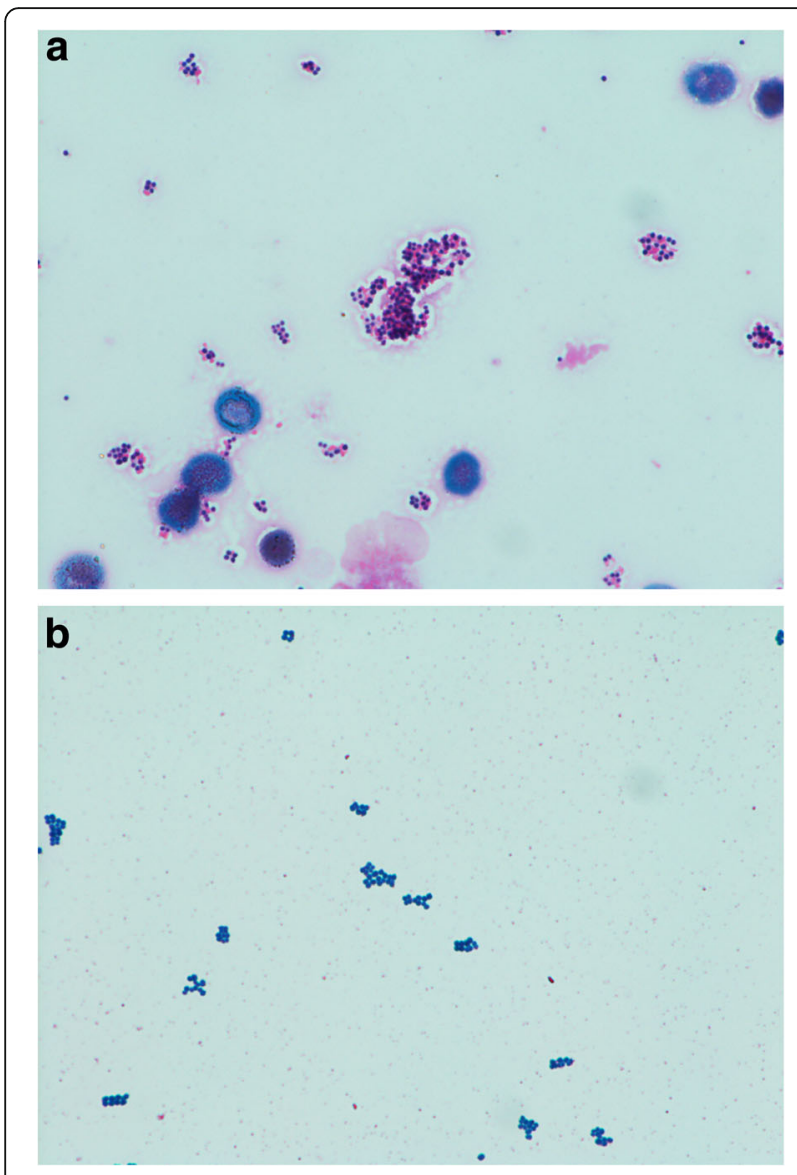

Fig. 1 Gram-stained smears from positive BacT/ALERT blood culture bottles showing Staphylococcus aureus and coagulase-negative staphylococci. (a) 'Oozing sign'-positive S. aureus (Gram-positive cocci in clusters with pink coloured 'oozing), aerobic bottles. (b)

'Oozing sign'-negative Staphylococcus epidermidis, aerobic bottles

with the presence of the 'oozing sign' to predict the identity of either $S$. aureus or other coagulase-negative staphylococci.

\section{Materials and methods}

This single-centre, prospective observational study was performed at St. Mary's Hospital (a 1,097-bed acute tertiary care teaching hospital in Fukuoka, Japan) from May 2017 to November 2017. We used routine blood culture bottles (BacT/ALERT FA and BacT/ALERT SN, bioMérieux, Inc., Tokyo, Japan). Bacterial species were identified and the minimum inhibitory concentrations were determined by using the MicroScan WalkAway 96 SI system (Beckman Coulter, Tokyo, Japan). Positive bottles were removed, and Gram staining was performed using Favor method (Nishioka's method) as described below [5]. Heat-fixed smears on slides were flooded with $0.2 \%$ Victoria blue for $1 \mathrm{~min}$ and then washed with tap water. Next, the smears were decolourized with $2 \%$ picric acid-ethanol, counterstained with $0.004 \%$ fuchsin for $1 \mathrm{~min}$, and finally washed with tap water. When the signal became positive between $5 \mathrm{pm}$ and $9 \mathrm{am}$, Gram staining was performed the next morning. The inclusion criteria were: (a) Gram-positive cocci in clusters and (b) an identification of Staphylococcus. The exclusion criteria were: (a) the identification of more than two species and (b) an identification of a species other than Staphylococcus. The examiners subjectively assessed Gramstained samples for the presence or absence of pink oozing surrounding the clustered Gram-positive cocci, the so-called 'oozing sign' (Fig. 1). The Gram staining was evaluated by two examiners who knew whether the Gram staining was from either aerobic or anaerobic blood cultures, but were blinded to the identification findings. We then analysed the diagnostic performance of Gram staining of the blood cultures. This study was approved by the St. Mary's Hospital Research Ethics Committee (Approval No. 17-0101). Blood culture samples were collected daily as part of standard patient care. Since this was an observational study, informed consent was waived.

\section{Statistical analysis}

Precision of the 'oozing sign' was evaluated by determining its sensitivity and specificity in comparison to culture-based results. Two test results from aerobic and anaerobic bottles were obtained from each patient, and the two 'oozing sign' test results were correlated. To assess the correlation, the generalized mixed model was employed (Additional file 1). Sensitivity and specificity can be modelled with the log link function [6]. Data analyses were carried out in two steps. First, the effect of the covariate was tested, namely the effect of the bottle. The second step was to estimate the sensitivity and specificity with/ without covariates. $P$ values less than 0.05 were considered statistically significant. All statistical analyses were performed with SAS (version 9.4; SAS Institute, Cary, NC).

\section{Results}

A total of 136 samples were screened for study inclusion. Based on the exclusion criteria, 13 samples with more than two species and five samples with species other than Staphylococcus, including Micrococcus sp. $(n=2)$, Enterococcus faecalis $(n=1), \alpha$-Streptococcus $s p .(\mathrm{n}=1)$, and Streptococcus parasanguis $(\mathrm{n}=1)$, were excluded. Finally, 118 bottle samples, including 55 aerobic and 63 anaerobic samples, were analysed. The results of the cultures were as follows: $50 \mathrm{~S}$. aureus (26 aerobic and 24 anaerobic samples) and 68 coagulase-negative staphylococci (39 aerobic and 29 anaerobic samples). There was $100 \%$ correlation between the aerobic and anaerobic bottles. The overall sensitivity of Gram staining was $78.7 \%$ (95\% CI: $65.8-94.3 \%$ ), and the specificity was $95.0 \%$ (95\% CI: 84.7-98.4\%). 


\section{Discussion}

To the best of our knowledge, this is the first study on the diagnostic performance of blood culture Gram staining for the detection of $S$. aureus focused on the 'oozing sign' using the BacT/ALERT blood culture system. In this study, this 'oozing sign' had high overall diagnostic performance, regardless of aerobic or anaerobic culture. This 'oozing sign' is a simple and easy method that discriminates between $S$. aureus and other bacterial strains. A previous study showed that criteria based on direct Gram staining characteristics from positive blood cultures were useful for distinguishing $S$. aureus from other staphylococci [7]. This previous study, which was focused on the findings of cell size and cluster characteristics, was able to distinguish between $S$. aureus and coagulase-negative staphylococci. The overall sensitivity was $89 \%$, and the specificity was $98 \%$ from BacT/ALERT blood culture bottles, which is the same type of bottles used in our study. For example, they found that $S$. aureus grew as small $(<1 \mu \mathrm{m}$ irregular clusters containing many bacteria in the anaerobic bottles and as large $(\geq$ $1 \mu \mathrm{m})$ clusters in the aerobic bottles. In contrast, our study just evaluated one simple finding, 'oozing, regardless of whether aerobic or anaerobic bottles were used. Our method does not require measurement of cell size, thus, it seems to be more simple, which we believe is advantageous. This method could be used in hospitals with limited resources and outside of Japan.

There are some limitations to this study. Firstly, the results of this study were based on a subjective assessment. In fact, approximately $10 \%$ of the assessments were not in agreement. In the future, examiners may need to look at the slides together to obtain the best agreement. Secondly, the findings of this study are applicable only when using the same blood culture bottles and system, as other blood culture bottles may not yield the same result. Thirdly, Gram staining of the positive bottles was not performed at night, and there is a possibility that the 'oozing sign' is a time-dependent finding. However, we did not take a possible relationship with time into account. Finally, we do not know what the 'oozing sign' is; therefore, further research is needed to characterize this 'oozing sign'.

\section{Conclusion}

In conclusion, the 'oozing sign' observed in Gram staining is useful for rapidly identifying $S$. aureus in BacT/ ALERT blood culture bottles. The sensitivity and specificity of this simple finding are relatively high. Therefore, laboratories using the BacT/ALERT blood culture system should learn this simple and easy discrimination method for the rapid identification of S. aureus bacteraemia.

\section{Additional file}

Additional file 1: Modelling sensitivity and specificity by the generalized mixed model. (DOCX $12 \mathrm{~kb})$

\section{Acknowledgements}

The authors thank the clinical laboratory centre staff at St. Mary's Hospital for their excellent work.

We would like to thank Editage (https://www.editage.jp) for English language editing.

Funding

The authors declare that they have no funding sources.

\section{Availability of data and materials}

The datasets used and/or analysed in the current study are available from the corresponding author upon request.

\section{Authors' contributions}

YH collected, analysed, summarized, and interpreted the data, and drafted the manuscript. MI and KI collected the data. TK analysed the data. All authors read and approved the final manuscript.

\section{Ethics approval and consent to participate}

This study was approved by the institutional review board of St. Mary's Hospital (No. 17-0101). The requirement for obtaining written consent from study participants was waived by the IRB because of the observational nature of the study without any deviation from current medical practices.

\section{Consent for publication}

Not applicable.

\section{Competing interests}

The authors declare that they have no competing interests.

\section{Publisher's Note}

Springer Nature remains neutral with regard to jurisdictional claims in published maps and institutional affiliations.

\section{Author details}

${ }^{1}$ Department of Infectious Diseases, St. Mary's Hospital, Kurume, Japan. ${ }^{2}$ Biostatistics Center, Kurume University School of Medicine, Kurume, Japan. ${ }^{3}$ Department of Clinical Laboratory, St. Mary's Hospital, Kurume, Japan.

Received: 22 June 2018 Accepted: 24 September 2018 Published online: 29 September 2018

\section{References}

1. van Hal SJ, Jensen SO, Vaska VL, Espedido BA, Paterson DL, Gosbell IB. Predictors of mortality in Staphylococcus aureus bacteremia. Clin Microbiol Rev 2012;25:362-386.

2. Khatib R, Johnson LB, Fakih MG, Riederer K, Khosrovaneh A. Shamse, et al. persistence in Staphylococcus aureus bacteremia: incidence, characteristics of patients and outcome. Scand J Infect Dis. 2006;38:7-14.

3. Idelevich EA, Schüle I, Grünastel B, Wüllenweber J, Peters G, Becker K. Rapid identification of microorganisms from positive blood cultures by MALDITOF mass spectrometry subsequent to very short-term incubation on solid medium. Clin Microbiol Infect. 2014;20:1001-6.

4. Lowy FD. Antimicrobial resistance: the example of Staphylococcus aureus. J Clin Invest. 2003;111:1265-73.

5. Tanaka N, Nagata N, Ooshige T, Takasaki, M. The usability of a simple Gram stain procedure (Nishioka's method). J J.jn Soc Intensive Care Med. 1997;4: 383 [In Japanese].

6. Margaret Sullivan Pepe. The statistical evaluation of medical tests for classification and prediction. In: Oxford University press; 2003.

7. Murdoch DR, Greenlees RL. Rapid identification of Staphylococcus aureus from BacT/ALERT blood culture bottles by direct gram stain characteristics. J Clin Pathol. 2004;57:199-201. 